\title{
ELEMENTARY PROOF OF A THEOREM ON CONFORMAL RIGIDITY ${ }^{1}$
}

\section{EDGAR REICH}

Let $f(z)$ be single valued and analytic in the annulus $A: a<|z|<b$, $f(A) \subset A$, and suppose that $f$ maps every closed curve with winding number +1 about $z=0$ onto a curve with the same property. Then $[1],[2],[3],[4] f$ if a rotation. Stimulated by a discussion with Professor A. Marden we shall give a short proof of this result with a minimum of topological notions.

By hypothesis, the integral of

$$
\frac{1}{z}-\frac{f^{\prime}(z)}{f(z)}
$$

around any closed curve in $A$ vanishes. Hence there exists a branch, $F(z)$, of $\log [z / f(z)]$ single valued in $A$. Let us define

$$
\begin{aligned}
u\left(r e^{i \theta}\right) & =\operatorname{Re} F\left(r e^{i \theta}\right)=\log r-\log \left|f\left(r e^{i \theta}\right)\right|, \quad a<r<b, \\
I(r) & =(2 \pi)^{-1} \int_{0}^{2 \pi} u\left(r e^{i \theta}\right) d \theta .
\end{aligned}
$$

By Cauchy's theorem applied to $z^{-1} F(z), I(r)$ is independent of $r$. On the other hand, since $f(A) \subset A$,

$$
\log (r / b) \leqq u\left(r e^{i \theta}\right) \leqq \log (r / a), \quad a<r<b .
$$

Therefore

$$
\limsup _{r \rightarrow a} I(r) \leqq 0, \quad \liminf _{r \rightarrow b} I(r) \geqq 0 .
$$

Thus, $I(r) \equiv 0, a<r<b$. Hence, by (1),

$$
\begin{aligned}
J(r) & =(2 \pi)^{-1} \int_{0}^{2 \pi}\left|u\left(r e^{i \theta}\right)\right| d \theta=(2 \pi)^{-1}\left[\int_{u \geqq 0} u d \theta-\int_{u \leqq 0} u d \theta\right] \\
& =-2(2 \pi)^{-1} \int_{u<0} u d \theta \leqq 2 \log (b / r),
\end{aligned}
$$

or alternatively,

Received by the editors April 20,1965.

${ }^{1}$ Work done with support from NSF grant GP 3904 with the University of Minnesota. 
(3)

$$
J(r)=2(2 \pi)^{-1} \int_{u>0} u d \theta \leqq 2 \log (r / a), \quad a<r<b .
$$

Since $\left|u\left(r e^{i \theta}\right)\right|$ is subharmonic, $J(r)$ is a convex function of $\log r$. Therefore $J(r) \leqq \max \left[J\left(r_{1}\right), J\left(r_{2}\right)\right]$ whenever $a<r_{1} \leqq r \leqq r_{2}<b$. Letting $r_{1} \rightarrow a, r_{2} \rightarrow b$, and using (2), (3) we obtain

$$
J(r) \equiv 0, \quad a<r<b .
$$

Thus $u \equiv 0,\left|z^{-1} f(z)\right| \equiv 1$. Thus $f(z)=e^{i c} z, c$ real.

\section{REFERENCES}

1. H. Huber, Über analytische Abbildungen von Ringgebieten in Ringgebieten, Compositio Math. 9 (1951), 161-168.

2. J. A. Jenkins, Some results related to extremal length, Ann. of Math. 30 (1953), 87-94.

3. H. J. Landau and R. Osserman, On analytic mappings of Riemann surfaces, J. Analyse Math. 7 (1959/60), 249-279.

4. M. Schiffer, On the modulus of doubly-connected domains, Quart. J. Math. Oxford Ser. 17 (1946), 197-213.

UNIVERSITY OF MiNNESOTA 